\title{
Effect of High Fructose-Induced Metabolic Syndrome on Tissue Vitamin E and Lipid Peroxide Levels in Rats
}

\author{
Akira KITAgAWA $^{1}$, Yoshiji OHTA ${ }^{2, *}$, Koji OHASHI ${ }^{3}$, Koji YASHIRO ${ }^{2}$ and Kenji FuKUZAWA ${ }^{4}$ \\ ${ }^{1}$ Department of Nutrition, Faculty of Wellness, Shigakkan University, Ohbu, Aichi 474-8651, Japan \\ ${ }^{2}$ Department of Chemistry, Fujita Health University School of Medicine, Toyoake, Aichi 470-1192, Japan \\ ${ }^{3}$ Department of Biomedical and Analytical Sciences, Fujita Health University School of \\ Medical Sciences, Toyoake, Aichi 470-1192, Japan \\ ${ }^{4}$ Department of Pharmacy, Faculty of Pharmacy, Yasuda Women's University, \\ Asaminami-ku, Hiroshima 731-0153, Japan \\ (Received October 30, 2019)
}

\begin{abstract}
Summary In the present study, we examined the effect of high fructose-induced metabolic syndrome (MetS) on tissue vitamin E and lipid peroxide (LPO) levels in rats. Feeding of a diet containing $60 \%$ fructose (HFD) to Wistar rats for 2, 4, and 6 wk caused week-dependent increases in HOMA-IR score and serum insulin, triglyceride, total cholesterol, and free fatty acid concentrations. Each week HFD feeding increased serum vitamin E concentration. Six-week HFD feeding reduced vitamin E status (the serum ratio of vitamin E/triglyceride+total cholesterol). Four- and 6-wk HFD feeding increased serum LPO concentration. Two-week HFD feeding increased liver, heart, kidney, and skeletal muscle (SM) vitamin E contents and decreased white adipose tissue (WAT) vitamin E content. Four- and 6-wk HFD feeding further reduced WAT vitamin E content without affecting the increased kidney and SM vitamin E contents. Six-week HFD feeding reduced the increased liver and heart vitamin E contents below the level of non-HFD feeding. Four-week HFD feeding increased heart and WAT LPO contents. Six-week HFD feeding increased liver LPO content and further increased heart and WAT LPO contents. Kidney and SM LPO contents remained unchanged. These results indicate that HFD-rats with early MetS have increased liver, kidney, heart, and SM vitamin E contents and decreased WAT vitamin E content under unchanged tissue LPO content and vitamin E status, while HFD-fed rats with progressed MetS have both decreased liver, heart, and WAT vitamin E contents under increased tissue LPO content and disrupted vitamin E status.
\end{abstract}

Key Words high fructose diet feeding, rat serum, rat tissues (liver, kidney, heart, skeletal muscle, and white adipose tissue), hyperinsulinemia, insulin resistance, dyslipidemia, hypertriglyceridemia, hypercholesterolemia, lipid peroxidation

Metabolic syndrome (MetS) has a high prevalence around the world. MetS is a cluster of symptoms with obesity, insulin resistance, dyslipidemia, hypertension, and hyperuricemia which leads to cardiovascular disease, type-2 diabetes, and non-alcoholic fatty liver (1). In addition, oxidative stress is involved in the pathogenesis of MetS $(2,3)$. High fructose intake has been shown to be associated with the rising prevalence of MetS, triggering function impairment in multiple tissues and organs $(4,5)$.

Obesity, insulin resistance, dyslipidemia, hypertension, and hyperuricemia are induced in rats with high fructose (HF) intake like the case of humans with $\mathrm{HF}$ intake $(6,7)$. Therefore, rats with HF intake have been used as a model of MetS. Vitamin E, a fat-soluble vitamin, functions as an antioxidant by breaking the chain reaction of lipid peroxidation induced by free radicals

\footnotetext{
*To whom correspondence should be addressed.

E-mail: yohta@fujita-hu.ac.jp
}

and by scavenging reactive oxygen species, especially singlet oxygen (8). It has been reported that vitamin $\mathrm{E}$ supplementation improves the free radical defense system potential and insulin sensitivity of rats fed a diet rich in fructose for $6 \mathrm{wk}$ (9). Thus, oxidative stress occurs in rats with HF-induced MetS as found in humans with MetS. There are some reports showing that a decrease in vitamin E level with an increase in lipid peroxide (LPO) level occurs in the liver, kidney, and heart of MetS rats fed a diet rich in fructose for 20-45 d (10-13). There is a report showing that a decrease in vitamin $\mathrm{E}$ level with an increase in LPO level occurs in the skeletal muscle (SM) of MetS rats fed a diet rich in fructose for $30 \mathrm{~d}$ (14). There is also a report showing that a decrease in vitamin E level with an increase in LPO level occurs in the abdominal adipose tissue of MetS rats given a drinking water rich in fructose for $3 \mathrm{wk}$ (15). Furthermore, it has been reported that vitamin $\mathrm{E}$ level decreases, increases or is unchanged with an increase in LPO level in the plasma of rats when MetS is induced by feeding a fruc- 

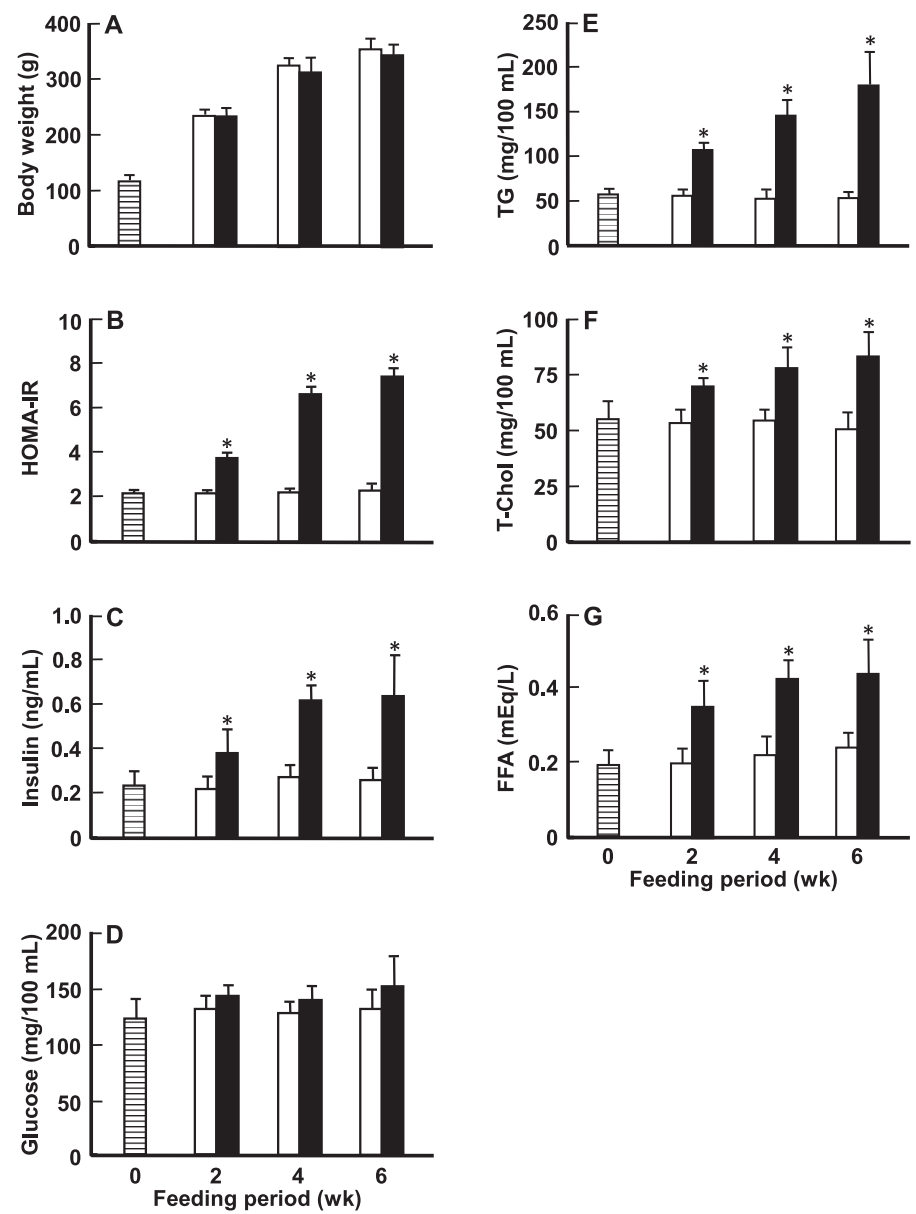

Fig. 1. Effects of 2-, 4-, and 6-wk HFD feeding on body weight (A), HOMA-IR score (B) and serum insulin (C), glucose (D), TG (E), T-Chol (F), and FFA (G) concentrations in rats. White columns indicate CD-fed rats. Black columns indicate HFD-fed rats. HFD feeding, body weight measurement, HOMA-IR determination, and serum insulin, glucose, and lipid assays were performed as described in "Materials and Methods." Results are means \pm SD ( $n=8$ for HFD-fed rats at each week HFD feeding; $n=5$ for CD-fed rats at each week HFD feeding). ${ }^{*} p<0.05$ (versus the corresponding CD-fed rats).

tose-enriched diet for $2-6 \mathrm{wk}(9,16-21)$. The serum or plasma ratio of vitamin $\mathrm{E}$ to lipids such as total cholesterol (T-Chol), triglyceride (TG) or T-Chol plus TG has been used to estimate vitamin E status in humans and experimental animals (22-25). It has been shown that the plasma ratio of vitamin $\mathrm{E}$ to triglyceride decreases or increases in MetS rats fed a diet rich in fructose for 2, 4 or $6 \mathrm{wk}(16,20,21)$. However, it is still unclear how the changes in tissue vitamin E contents occurs following the development of HF-induced MetS in rats. It is also unclear at present whether the changes in tissue vitamin E levels following the development of HF-induced MetS are associated with the changes in the corresponding tissue LPO levels and vitamin E status in rats.

In the present study, therefore, we examined the effect of HF-induced MetS on vitamin E and LPO levels in the liver, kidney, heart, SM, and adipose tissue of rats. Furthermore, we examined how vitamin E status estimated by the serum ratio of vitamin E to TG plus T-Chol changes with the development of HF-induced MetS in rats.

\section{Materials and Methods}

Chemicals. Ethylenediaminetetraacetic acid (EDTA), tetramethoxypropane, 2-thiobarbituric acid (TBA), $\alpha$ tocopherol $(\alpha$-Toc) and $\delta$-tocopherol used for vitamin E determination, and other chemicals were purchased from FUJIFILM Wako Pure Chemical Corporation (Osaka, Japan). All chemicals were of regent grade and were not further purified.

Animals. Male Wistar rats aged 4 wk were purchased from Japan SLC, Inc. (Hamamatsu, Japan). The animals were maintained under a daily controlled lighting cycle (12 h-light and $12 \mathrm{~h}$-dark cycling) at $23^{\circ} \mathrm{C}$ and $50 \%$ humidity with free access to Oriental MF rat chow (Oriental Yeast Co., Ltd., Tokyo, Japan) and water for $1 \mathrm{wk}$ prior to the experiment. All animals received humane care in compliance with the guidelines of the Management of Laboratory Animals in Fujita Health University. The animal experiment was approved by Institutional Animal Care and Use Committee. The approved protocol number was H0821.

Diet feeding and sample collection. Five-week-old rats were fed either a control diet (CD) or a high-fructose diet (HFD) for 2, 4, and 6 wk. The composition of CD was $60 \%$ dextrose, $20 \%$ milk casein, $5.2 \%$ soy oil, $3.5 \%$ mineral mixture, $1.0 \%$ vitamin mixture, $10.05 \%$ cellulose powder, $0.2 \%$ choline bitartrate, and $0.00104 \%$ 
tert-butyl hydroquinone. The composition of HFD was the same except that $60 \%$ dextrose was replaced by $60 \%$ fructose. Both CD and HFD were supplied by Oriental Yeast Co., Ltd. CD and HFD were pair-fed to ensure the same daily calorie intake in rats fed CD and HFD. After 2-, 4- or 6-wk HFD feeding, rats fasted for $5 \mathrm{~h}$ were weighed and then sacrificed under anesthesia with sodium pentobarbital. At which time blood was collected from the inferior vena cava. Serum was obtained from the collected blood by centrifugation. Immediately after sacrifice, perfusion through the portal vein was conducted with ice-cold $0.9 \% \mathrm{NaCl}$ to remove residual blood in tissues. After perfusion, the liver, kidney, and heart were isolated from each rat. The skeletal muscle (SM) and white adipose tissue (WAT) were also obtained from the biceps femoris muscle and periepididymal fat pad, respectively, of each rat. These tissues were washed with ice-cold $0.9 \% \mathrm{NaCl}$, wiped on a paper filter, and then weighed. The collected serum and tissues were stored at $-80^{\circ} \mathrm{C}$ until use.

Biochemical determinations. Serum insulin was assayed using Rat insulin ELISA kit (Mereodia, Uppsala, Sweden). Serum glucose, TG, T-Chol, and free fatty acids (FFA) were assayed using commercial kits, Glucose CIITest Wako, Triglyceride R-Test Wako, Cholesterol E-Test Wako, and NEFA C-Test Wako (FUJIFILM Wako Pure Chemical Corporation), respectively. The determined fasting serum glucose and insulin concentrations were used for the assessment of insulin resistance using homeostatic model assessment insulin resistance (HOMA-IR) score. HOMA-IR was calculated with the formula [fasting serum insulin $(\mu \mathrm{U} / \mathrm{mL}) \times$ fasting serum glucose $(\mathrm{mM}) /$ 22.5], as described by Matthews et al. (26). Serum LPO was measured by the fluorometric TBA method (27) using tetramethoxypropane as a standard. The concentration of serum LPO is expressed as the amount of malondialdehyde (MDA) equivalents. Each tissue was homogenized in nine volume of ice-cold $50 \mathrm{~mm}$ Tris-HCl buffer ( $\mathrm{pH} 7.4$ ) containing $1 \mathrm{~mm}$ EDTA to prepare 10\% homogenate. LPO in each tissue homogenate was assayed by the colorimetric TBA method (28) using tetramethoxypropane as a standard except that the reaction mixture contained $1 \mathrm{~mm}$ EDTA. The amount of tissue LPO is expressed as the amount of MDA equivalents. $\alpha$ Tocopherol in serum and each tissue homogenate was assayed by the high-performance liquid chromatographic method with electrochemical detection using $\delta$-tocopherol as an internal standard as described in our previous report (29). The amount of vitamin $\mathrm{E}$ in serum and each tissue homogenate is expressed as that of $\alpha$-Toc.

Statistical analysis. All results obtained are expressed as the mean \pm SD. The statistical analyses of the results were performed using a commercial Excel statistical package (Microsoft Excel 2016). Each mean value was compared by one-way analysis of variance (ANOVA) and Bonferroni/Dunn for multiple comparisons. Associations between vitamin E and LPO levels in the serum and tissues of HFD-fed rats were assessed using Spearman rank correlation test. The significance level was set at $p<0.05$.
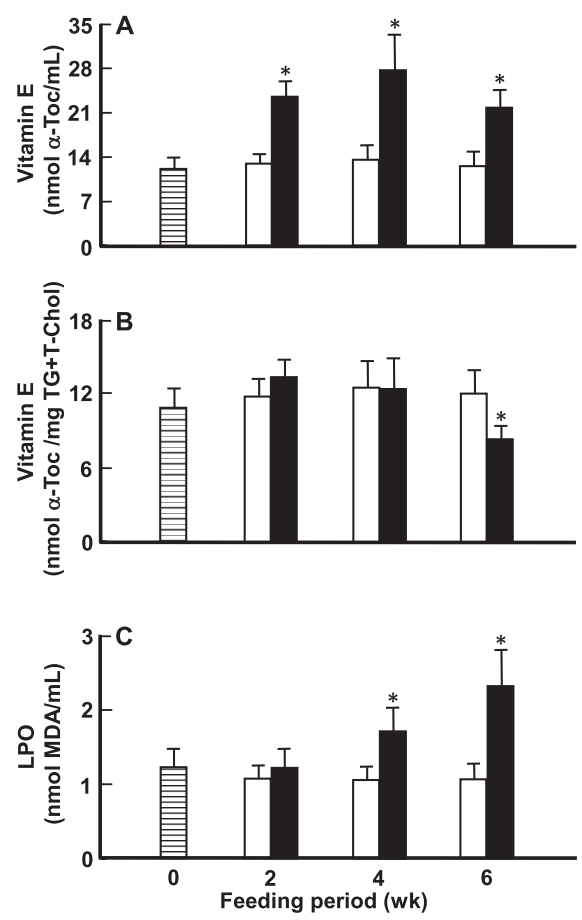

Fig. 2. Effects of 2-, 4-, and 6-wk HFD feeding on serum vitamin E (A), serum relative vitamin E (B), and LPO (C) concentrations in rats. White columns indicate CD-fed rats. Black columns indicate HFD-fed rats. HFD feeding, serum vitamin E and LPO assays, and serum relative vitamin $\mathrm{E}$ (the serum ratio of $\alpha$-Toc/TG + T-Chol) determination were performed as described in "Materials and Methods." Results are means \pm SD ( $n=8$ for HFDfed rats at each week HFD feeding; $n=5$ for CD-fed rats at each week HFD feeding). ${ }^{*} p<0.05$ (versus the corresponding $\mathrm{CD}$-fed rats).

\section{Results}

As shown in Fig. 1A, there was no significant difference in body weight used as an indicator of obesity between rats fed HFD for 2, 4, and $6 \mathrm{wk}$ and the corresponding rats fed CD. HOMA-IR score used as an indicator of insulin resistance and serum insulin, TG, T-Chol, and FFA concentrations in HFD-fed rats increased significantly at 2-wk HFD feeding when compared with those in CD-fed rats and these increases further progressed at 4- and 6-wk HFD feeding (Fig. 1B, C, E, F, and $\mathrm{G})$. However, there was no significant difference in serum glucose concentration between HFD- and CD-fed rats at each week HFD feeding (Fig. 1C). Thus, HFD-fed rats showed the apparent features of MetS such as insulin resistance, hyperinsulinemia, and dyslipidemia in a feeding week-dependent manner.

Serum vitamin E concentration was significantly higher in HFD-fed rats than in CD-fed rats at 2-, 4-, and 6-wk HFD feeding (Fig. 2A), while the level of serum relative vitamin E, i.e., the serum ratio of $\alpha$-Toc/TG $+\mathrm{T}-$ Chol, an index of vitamin E status, was significantly lower in HFD-fed rats than in ND-fed rats at 6-wk HFD feeding (Fig. 2B). Serum LPO concentration was significantly higher in HFD-fed rats than in CD-fed rats at 4and 6-wk HFD feeding and the increase in serum LPO 

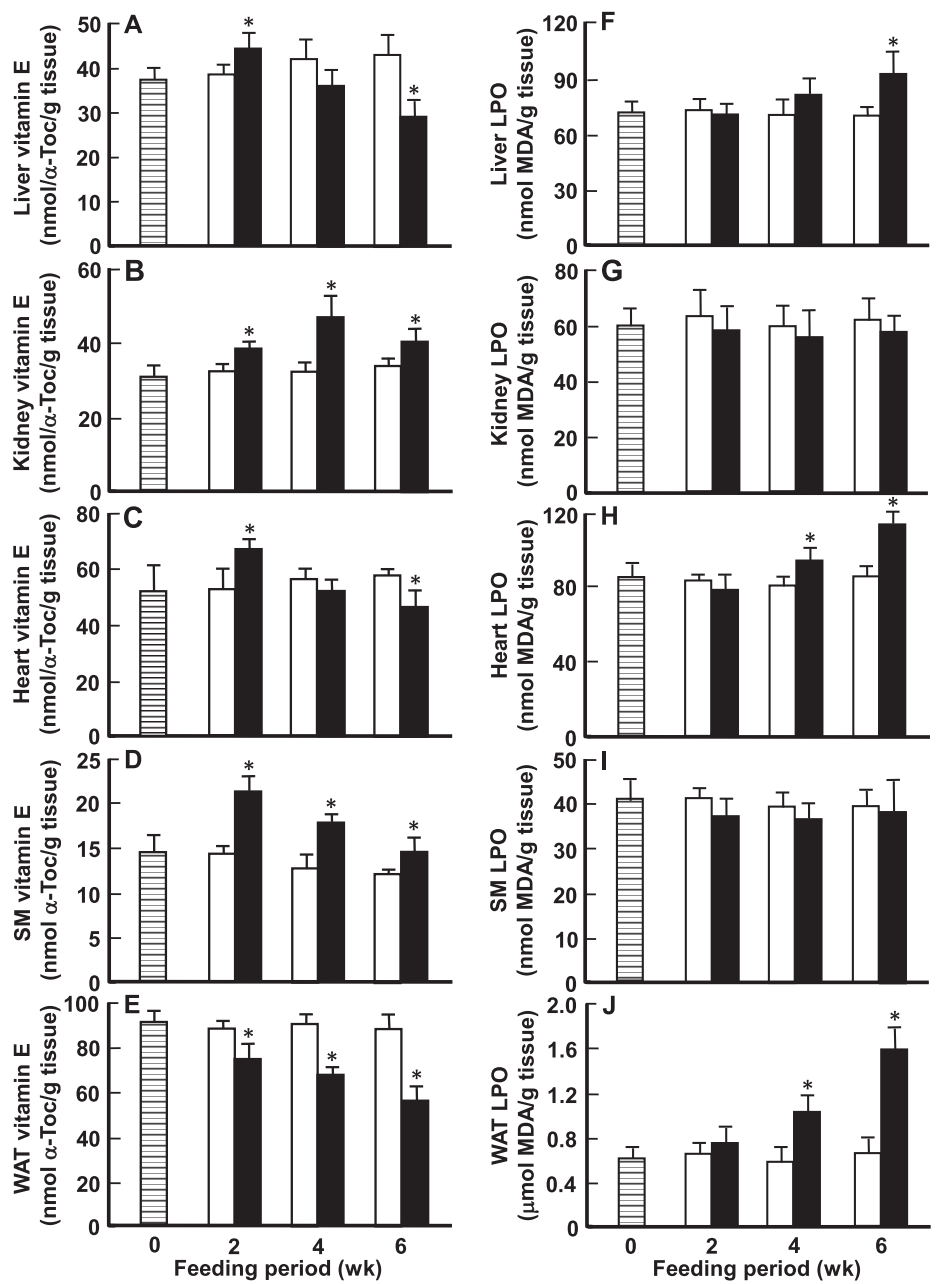

Fig. 3. Effects of 2-, 4-, and 6-wk HFD feeding on liver (A), kidney (B), heart (C), SM (D), and WAT (E) vitamin E contents and liver (F), kidney (G), heart (H), SM (I), and WAT (J) LPO contents in rats. White columns indicate CD-fed rats. Black columns indicate HFD-fed rats. HFD feeding and tissue vitamin E and LPO assays were performed as described in "Materials and Methods." Results are means \pm SD ( $n=8$ for HFD-fed rats at each week HFD feeding; $n=5$ for CD-fed rats at each week HFD feeding). ${ }^{*} p<0.05$ (versus the corresponding CD-fed rats).

concentration was higher at 6-wk HFD feeding than at 4-wk HFD feeding (Fig. 2C).

As shown in Fig. 3A, B, C, and D, liver, kidney, heart, and SM vitamin $\mathrm{E}$ contents in HFD-fed rats were significantly higher than those in CD-fed rats at 2-wk HFD feeding, while WAT vitamin E content was significantly lower in HFD-fed rats than in CD-fed rats at the same feeding period (Fig. 3E). There were no differences in liver and heart vitamin E contents between HFD- and CD-fed rats at 4-wk HFD feeding, but these contents were significantly lower in HFD-fed rats than in CD-fed rats at 6-wk HFD feeding (Fig. 3A and C). Kidney vitamin E content was significantly higher in HFD-fed rats than in ND-fed rats at 4- and 6-wk HFD feeding, although the increased vitamin E content at 4-wk HFD feeding was significantly higher at 2 -wk HFD feeding $(p<0.05)$ but the increased vitamin $\mathrm{E}$ content at 6 -wk HFD feeding tended to be lower that at 4-wk HFD feeding (Fig. 3B). SM vitamin E content in HFD-fed rats was significantly higher than that in CD-fed rats at 4- and 6-wk HFD feeding (Fig. 3D). However, the content of vitamin E in the SM of HFD-fed rats was significantly smaller at 4-wk HFD feeding than at 2-wk HFD feeding $(p<0.05)$ and at 6 -wk HFD feeding that at 4 -wk HFD feeding $(p<0.05)$ (Fig. 3D). WAT vitamin E content was significantly lower in HFD-fed rats that in CD-fed rats at 2-, 4-, and 6-wk HFD feeding and the WAT vitamin E content in HFD-fed rats dropped in a feeding week-dependent manner (Fig. 3E).

Liver LPO content in HFD-fed rats tended to be higher than that in CD-fed rats at 4-wk feeding and that content was significantly higher in HFD-fed rats than in CDfed rats at 6-wk HFD feeding (Fig. 3F). There were no significant differences in kidney and SM LPO contents between HFD-fed rats and CD-fed rats at each week HFD feeding (Fig. 3G and I). Heart and WAT LPO contents were significantly higher in HFD-fed rats than in CD-fed rats at 4- and 6-wk HFD feeding and the increases in heart and WAT LPO contents were significantly larger at 6 -wk HFD feeding than at 4 -wk HFD feeding $(p<0.05)$ (Fig. 3H and J).

To clarify whether and how the change in vitamin E level is associated with the change in LPO level in the serum and liver, kidney, heart, SM, and adipose tissues of rats fed HFD over $6 \mathrm{wk}$, the correlation of vita- 
min E level with LPO level was assessed in the serum and these tissues. As a result, there was no significant correlation of vitamin E level with LPO level in the serum $(r=0.2409, p>0.05)$ and kidney $(r=0.0533$, $p>0.05)$ and SM $(r=0.2620, p>0.05)$. In contrast, vitamin E level was significantly and highly correlated with LPO level in the liver $(r=-0.8359, p<0.05)$, heart $(r=-0.9722, p<0.05)$, and WAT $(r=-0.8015$, $p<0.05)$ in a negative manner.

\section{Discussion}

In the present study, although rats fed HFD, i.e., a diet containing $60 \%$ fructose, over $6 \mathrm{wk}$ showed no overweight as reported previously $(6,9,12,13,15-18,20$, 21), MetS in rats fed HFD appeared at 2-wk HFD feeding and progressed with an increase in HFD feeding period, judging from the MetS-related features such as insulin resistance (based on HOMR-IR score), hyperinsulinemia, and dyslipidemia associated with increases in serum TG, T-Chol, and FFA concentrations. Insulin resistance, hyperinsulinemia, and dyslipidemia found in rats fed HFD over $6 \mathrm{wk}$ have been observed in previous reports $(6,9-11,13-15,18,20,21)$. However, HFDfed rats showed no hyperglycemia at each week HFD feeding. As to glycemic status in rats fed a diet rich in fructose for $2-6 \mathrm{wk}$, there are several reports showing no hyperglycemia $(6,9,13,15,16,19-22)$, while there are several reports showing hyperglycemia $(10,11,17$, 18). However, the reason of the controversy regarding glycemic status in rats fed a diet rich in fructose over $6 \mathrm{wk}$ among these reports including our present report is unclear at present.

An increase in plasma vitamin E concentration has been reported in rats fed a diet containing 58\% fructose for 2 or $4 \mathrm{wk}(19,20)$, although there are some reports showing a decrease in plasma vitamin E concentration in rats fed a diet containing $60 \%$ fructose for $20-42 \mathrm{~d}$ $(10,11,17,18)$. In the present study, HFD-fed rats showed an increase in serum vitamin $\mathrm{E}$ concentration at an early stage of MetS, i.e., 2-wk HFD feeding. The increase in serum vitamin $\mathrm{E}$ concentration was maintained during the progression of MetS, i.e., 4- and 6-wk HFD feeding. Thus, no reduction of serum vitamin E concentration was observed in rats fed HFD for 2-6 wk. This finding allow us to assume the release of vitamin $\mathrm{E}$ from the liver, SM, and/or adipose tissue of HFD-fed rats into the blood during the development of MetS, because about $90 \%$ of total body mass of vitamin $\mathrm{E}$ is stored in the liver, SM, and adipose tissue of rats (30).

There are some reports showing that 4-wk feeding of a diet containing $58 \%$ fructose to rats causes an increase in the plasma ratio of vitamin E/triglyceride (20) and that 2- or 4-wk feeding of a diet containing 65\% fructose to rats causes a decrease in the plasma ratio of vitamin E/triglyceride $(16,21)$. The plasma ratio of vitamin $\mathrm{E}$ to lipids has been used to estimate vitamin E status in the body (23-26). In the present study, the level of serum relative vitamin E, i.e., the serum ratio of vitamin $\mathrm{E} / \mathrm{TG}+\mathrm{T}-\mathrm{Chol}$ used as an index of vitamin $\mathrm{E}$ status, in HFD-fed rats was lower than that in rats fed CD at 6-wk
HFD feeding. Thus, vitamin E status was found to be disrupted in HFD-fed rats with progressed MetS. This finding suggests that vitamin E consumption could occur in the tissues of HFD-fed rats with progressed MetS.

An increase in plasma LPO concentration has been reported in rats fed a diet containing $58 \%$ fructose for 2,4 or 6 wk $(9,19,20)$, in rats fed a diet containing $60 \%$ fructose for 20 or $30 \mathrm{~d}(10,11)$, in rats fed a diet containing $61 \%$ fructose for $20 \mathrm{~d}(18)$, and in rats given a drinking water containing 10\% fructose for $3 \mathrm{wk}(15)$. In the present study, 4- or 6-wk HFD feeding, but not 2-wk HFD feeding, to rats caused an increase in serum LPO concentration. The increased serum LPO concentration in HFD-fed was higher at 6 -wk feeding than at 4-wk feeding. These results suggest that systemic oxidative stress could develop with the progression of MetS in HFD-fed rats.

It has been reported that liver, kidney, heart, SM, and adipose tissue vitamin $\mathrm{E}$ contents decrease in rats fed a diet rich in fructose or given a drinking water rich in fructose for 2-6 wk (10-15). In the present study, increases in liver, kidney, heart and SM vitamin E contents and a decrease in WAT vitamin E content were found to occur in rats fed HFD for $2 \mathrm{wk}$, i.e., in HFD-fed rats with early MetS. As described above, the increase in serum vitamin $\mathrm{E}$ concentration was found in rats fed HFD for $2 \mathrm{wk}$. Adipose tissues are known to function as the storage of vitamin E in the body (31). Accordingly, these findings allow us to suggest that the increase in vitamin E level in the liver, kidney, heart and SM of HFDfed rats with early MetS could be due to the release of vitamin $\mathrm{E}$ from the WAT into the blood and the uptake of the released vitamin E by the liver, kidney, heart and SM. The increased liver and heart vitamin E contents found in HFD-fed rats with early MetS were reduced below the level of CD-fed rats at a progressed stage of MetS. The decrease in WAT vitamin E content in HFD-fed rats with early MetS was further enhanced at a progressed stage of MetS. However, the increased vitamin E content in the kidney and HFDF-fed rats with early MetS was maintained at a progressed stage of MetS. The increased vitamin E content in the SM of HFD-fed rats with early MetS was also maintained at a progressed stage of MetS, although the increased SM vitamin E content was reduced gradually with the progression of MetS. Thus, the change in vitamin E content in the tissues of HFD-fed rats with MetS was found to occur in a tissuedependent manner. Therefore, it can be thought that the decrease in vitamin E content in the liver, heart, and WAT of HFD-fed rats with progressed MetS contributes to the above-described disruption of vitamin E status. It has been shown in genetically obese rats that a marked increase in plasma vitamin E concentration occurs with a decrease in adipose tissue vitamin E content under hyperlipidemic conditions associated with increases in the concentrations of plasma total lipids and cholesterol (32). From this finding, it is suggested that the abovedescribed increase in serum vitamin E concentration in HFD-fed rats with developing MetS could be due to the release of vitamin $\mathrm{E}$ from the WAT under hyperlipidemic 
conditions associated with increases in serum TG and T-Chol concentrations.

There are some reports showing that an increase in LPO level with a decrease in vitamin E level occurs in the liver, heart, and kidney of rats fed a diet containing $60 \%$ fructose for 3-6 wk (10-13), in the liver, kidney, and SM of rats fed a diet containing $60 \%$ fructose for $30 \mathrm{~d}$ (14), and in the abdominal adipose tissue of rats given a drinking water containing $10 \%$ fructose for $3 \mathrm{wk}$ (15). In the present study, 2-wk HFD feeding to rats did not affect LPO content in the liver, kidney, heart, SM, and WAT. As described above, rats fed HFD for $2 \mathrm{wk}$ had unchanged serum LPO concentration and increased serum vitamin E concentration. These results may allow us to suggest that the above-described increase in vitamin E content in the liver, kidney, heart, and SM of rats fed HFD for 2 wk could be due to the release of vitamin E into the blood from the WAT, the major vitamin E-storing tissue in the body (32). Four-HFD feeding caused an increase in LPO content in the WAT and 6-wk HFD feeding caused further increase in LPO content in the tissue. Six-week HFD feeding caused an increase in LPO content in the liver and heart. However, 4- and 6-wk HFD feeding had no effect on LPO content in the kidney and SM. Thus, the above-described reduction of vitamin E content in the liver, heart, and WAT of HFD-fed rats with progressed MetS was found to occur in association with the increase in LPO content in these tissues. Furthermore, vitamin E content was highly correlated with LPO content in the liver, heart, and WAT of rats fed HFD over $6 \mathrm{wk}$ in a negative manner when the correlation of vitamin E content with LPO content was assessed in these tissues. Thus, a high reverse association of the reduction of vitamin $\mathrm{E}$ content with the increase in LPO content was confirmed in the liver, heart, and WAT of HFD-fed rats with developing MetS. As described above, rats fed HFD for 4 and $6 \mathrm{wk}$ had increased serum LPO concentration with increased serum vitamin E concentration. These results may allow us to suggest that the above-described decrease in vitamin E content in the liver and heart of HFD-fed rats with progressed MetS could be due to the consumption of vitamin E under enhanced lipid peroxidation in these tissues and that the reduction of vitamin E content in the WAT could be due to both the release of vitamin $\mathrm{E}$ into the blood from the tissue and the consumption of vitamin E under enhanced lipid peroxidation in the tissue. Furthermore, it is possible to suggest that the above-described disruption of vitamin E status in HFD-fed rats with progressed MetS could be due to the consumption of vitamin E under enhanced lipid peroxidation in the liver, heart, and/or WAT. It is also possible to suggest that enhanced lipid peroxidation in the liver, heart, and WAT of HFD-fed rats with progressed MetS could be relevant to systemic oxidative stress. However, it is unknown at present whether or how HFD feeding to rats over 6 wk affects the uptake of vitamin E by the tissues studied. Furthermore, vitamin E exerts intracellular antioxidant function depending on not only the vitamin itself but also other intercellular antioxidants such as vitamin $\mathrm{C}$ and reduced gluta- thione (GSH) (8). It has been reported that feeding of a diet rich in fructose to rats for $6 \mathrm{wk}$ causes not only a decrease in vitamin $\mathrm{E}$ level but also decreases in vitamin $\mathrm{C}$ and reduced glutathione levels in the liver and heart (10-12). Therefore, further studies are needed to elucidate the mechanism by which changes in tissue vitamin E levels in association with enhanced lipid peroxidation and the disruption of vitamin E status occur in HFD-fed rats with developing MetS.

In conclusion, the results of the present study indicate that HFD-fed rats with early MetS have increased liver, heart, kidney, and SM vitamin E contents and decreased WAT vitamin E content under unchanged tissue LPO content and vitamin E status, while HFD-fed rats with progressed MetS have both decreased liver, heart, and WAT vitamin E contents under increased tissue LPO content and disrupted vitamin E status.

\section{Disclosure of state of COI \\ No conflicts of interest to be declared.}

\section{REFERENCES}

1) Eckel RH, Grundy SM, Zimmert PZ. 2005. The metabolic syndrome. Lancet 365: 1415-1428.

2) Palmieri VO, Grattagliano I, Portinacasa F, Palciano G. 2006. Systemic oxidative alteration are associated with visceral adiposity and liver steatosis in patients with metabolic syndrome. J Nutr 136: 3022-3026.

3) Francisqueti FV, Chiaverini LC, Santos KC, Minatel IO, Ronchi CB, Ferron AJ, Ferreira AL, Corrêa CR. 2017. The role of oxidative stress on the pathophysiology of metabolic syndrome. Rev Assoc Bras 63: 185-191.

4) Miller A, Adeli K. 2008. Dietary fructose and the metabolic syndrome. Curr Opin Gastroenterol 24: 204-209.

5) Kleishadi R, Mansourian M, Heidari-Beni M. 2014. Association of fructose consumption and components of metabolic syndrome in human studies: a systematic review and meta-analysis. Nutrition 30: 503-510.

6) de Moura RF, Ribeiro C, de Oliveira JA, Stevanato E, de Mello MA. 2009. Metabolic syndrome signs in Wistar rats submitted to different high-fructose ingestion protocol. Br J Nutr 101: 1178-1184.

7) Tran LT, Yuen VG, McNeill JH. 2009. The fructose-fed rats: a review on the mechanisms of fructose-induced insulin resistance and hypertension. Mol Cell Biochem 332: $145-159$.

8) Niki E. 2014. Role of vitamin E as a lipid-soluble peroxyl radical scavenger: in vitro and in vivo evidence. Free Radic Biol Med 66: 3-12.

9) Faure P, Rossini E, Lafond JL, Richard MJ, Favier A, Halimi S. 1997. Vitamin E improves the free radical defense system potential and insulin sensitivity of rats fed high fructose diets. J Nutr 127: 103-107.

10) Thirunavukkarasu V, Nandhini ATA, Anuradha CV. 2004. Cardiac lipids and antioxidant status in high fructose rats and the effect of $\alpha$-lipoic acid. Nutr Metab Cardiovasc Dis 14: 351-357.

11) Nandhini ATA, Thirunavukkarasu V, Ravichandran MK, Anuradha CV. 2005. Effect of taurine on biomarkers of oxidative stress in tissues of fructose-fed insulin-resistant rats. Singapore Med J 46: 82-87.

12) Suganthi R, Rajamani $S$, Ravichandran MK, Anuradha CV. 2007. Effect of food seasoning spices mixture on 
biomarkers of oxidative stress in tissues of fructose-fed insulin-resistant rats. J Med Food 10: 149-153.

13) Ackerman Z, Oron-Herman M, Pappo O, Plelg E, Safadi R, Schmilovitz-Weiss H, Grozovski M. 2010. Hepatic effect of rosiglitazone in rats with the metabolic syndrome. Basic Clin Pharmacol Toxicol 107: 663-668.

14) Rajasekar P, Anuradha CV. 2007. Effect of L-carnitine on skeletal muscle lipids and oxidative stress in rats fed high-fructose diet. Exp Diabetes Res 2007: 72741.

15) Fariña JP, García ME, Alzamendi A, Giovambatista A, Marra CA, Spined E, Gagliardino JJ. 2013. Antioxidant treatment prevents the development of fructose-induced abdominal adipose tissue dysfunction. Clin Sci 126: 87-97.

16) Busserolles J, Gueux E, Rock E, Mazur A, Rayssiguier Y. 2002. Substituting honey for refined carbonhydrates protects rats from hypertriglyceridemic and peroxidative effects of fructose. J Nutr 132: 3379-3382.

17) Srividhya S, Ravichandran MK, Anuradha CV. 2002. Metoformin attenuates blood lipid peroxidation and potentiates antioxidant defense in high fructose-fed rats. J Biochem Mol Biol Biophys 6: 379-385.

18) Thirunavukkarasu V, Anuradha CV. 2004. Influence of $\alpha$-lipoic acid on lipid peroxidation and antioxidant defence system in blood of insulin-resistant rats. Diabetes Obes Metab 6: 200-207.

19) Faure P, Barclay D, Joyeux-Faure M, Halimi S. 2007. Comparison of the effects of zinc alone and zinc associated with selenium and vitamin $\mathrm{E}$ on insulin sensitivity and oxidative stress in high-fructose-fed rats. J Trace Elem Med Biol 21: 113-119.

20) Joyeux-Faure M, Rossini E, Ribuot C, Faure P. 2006. Fructose-fed rat hearts are protected against ischemiareperfusion injury. Exp Biol Med 231: 456-462.

21) Busserolles J, Gueux E, Rock E, Demigné C, Mazur A, Rayssiguier Y. 2003. Oligofructose protects against the hypertriglyceridemia and pro-oxidative effects of high fructose diet in rats. J Nutr 133: 1903-1908.

22) Thurmham DI, Davies JA, Crump BJ, Situnayake RD, Davis M. 1986. The use of different lipids to express serum tocopherol: lipid ratios for the measurement of vitamin E status. Ann Clin Biochem 23: 514-520.

23) Ford L, Farr J, Morris P, Berg J. 2006. The value of measuring serum cholesterol-adjusted vitamin E in routine practice. Ann Clin Biochem 43: 130-134.

24) Kaite M, Lebold BS, Grant KA, Freeman WM, Wiren KM, Miller GW, Kiley C, Leonard SW, Traber MG. 2011. Individual differences in hyperlipidemia and vitamin $\mathrm{E}$ status in response to chronic alcohol self-administration in cynomolgus monkeys. Alcohol Clin Exp Res 35: 474-483.

25) Takitani K, Inoue K, Koh M, Miyazaki H, Ishi K, Inoue A, Tami H. 2014. $\alpha$-Tocopherol status and altered expression of $\alpha$-tocopherol-related proteins in spreptozotocininduced type 1 diabetes in rat models. J Nutr Sci Vitaminol 60: 380-386.

26) Matthews DR, Hosker JP, Rudenski AS, Naylor BA, Treacher DF, Turner RC. 1985. Homeostasis model assessment: insulin and beta-cell function from fasting plasma glucose and insulin concentration in man. Diabetologia 28: 412-419.

27) Yagi K. 1975. A simple fluorometric assay for lipoperoxides in blood sample. Biochem Med 15: 212-216.

28) Ohkawa H, Ohishi N, Yagi K. 1979. Assay for lipid peroxides in animal tissues by thiobarbituric acid reaction. Anal Biochem 95: 351-358.

29) Kamiya Y, Ohta Y, Imai Y, Arisawa T, Nakano H. 2005. A critical role of gastric ascorbic acid in the progression of acute gastric mucosal lesions induced by compound 48/80 in rats. World J Gastroenterol 11: 1324-1332.

30) Bjørneboe A, Bjørneboe GE, Drevon CA. 1990. Absorption, transport and metabolism of vitamin E. J Nutr 120: 233-242.

31) Landrier J-F, Marcotorchino J, Tourniaire F. 2012. Lipophilic micronutrients and adipose tissue biology. Nutrients 4: 1622-1649.

32) Bieri JG, Evarts P. 1975. Effect of plasma lipid levels and obesity on tissue stores of $\alpha$-tocopherol. Proc Soc Exp Biol Med 149: 500-502. 